\title{
The Analysis of Cultural Gaps in Translation and Solutions
}

\author{
Jianqing $\mathrm{Wu}$ \\ Foreign languages college \\ Qingdao University of Science and Technology \\ Qingdao 266061, China \\ Email:wjq58@163.com
}

\begin{abstract}
This paper is about cultural gaps and their translation. It compares the different cultural connotations of expressions in English and Chinese. First, it analyzes the following cases which are often found in translation: cultural background; non-equivalence; extension and intension; derivation. Then six methods are put forward to solve those above problems.
\end{abstract}

Keywords: Culture background, Non-equivalence, Extension and intension, Derivation

\section{Introduction}

Translation, the act or process of rendering what is expressed in one language or set of symbols by means of another language or set of symbols. It has for centuries been taken for granted that translation takes place between languages. Undoubtedly, as an important communication way, one of the basic principles of translation is to be faithful to the original. According to this principle, translation should first be faithful to the content of original, with literal translation conveying the original cultural connotation in a precise way. Maybe cultural gaps are an obstacle when we do translation, but in my opinion, everything is translatable. Because translation is a "re-coding" or a change of surface structure in representation of the deep structure underlying it.

\section{Cultural Gaps Reflected in Translating Process}

We all know that each nationality has its own culture. It's the unique symbol of their mind. A society's culture consists of whatever it is one has to know or believe in order to operate in a manner acceptable to its members, and do so in any role that they accept for any one of themselves. In a word, culture refers to the entire way of life of a society. It includes not only material things such as cities, organizations, and schools, but also nonmaterial things such as ideas, customs, family patterns and languages. Language is not seen as an isolated phenomenon suspended in a vacuum but as an integral part of culture. Some social scientists consider it the keystone of culture. On the other hand, language is influenced and shaped by culture. It reflects culture. It just likes a bridge between culture and translation, so it plays a very important role in it.

As we know, during translating process, cultural differences often make us feel awkward. How can we deal with it properly? In my view, first of all we should see through to the essence of this circumstance. As the word is the most active and the most lively element of a language, the changes in social life and the development in national culture can be reflected in words quickly and directly. So we must pay more attention to the analysis and comparison of the cultural connotation of words for it reflects the cultural differences in different countries.

And these cultural gaps can be found in the following areas: cultural background, non-equivalence, extension and intention, and derivation.

\subsection{Cultural Background}

When you are communicating with somebody from your own culture, you can express yourself or represent your experience much easier because you can share many similar ideas with him. But when communication is between people from different culture, the process is more troublesome. Words, as an effective tool, reflect author's attitude and beliefs, their points of view. And we can say, language expresses cultural reality.

So intercultural awareness becomes especially important. When you encounter a new language some things are easy to learn. You just patch on some new lexical items and grammatical forms and continue listening and talking. Other things are more difficult, though with a little effort the differences from one language to another can be bridged. Lack of cultural knowledge affects our comprehension negatively. Maybe someone thinks intercultural awareness can grow naturally, but I think it has to be trained. For example, when a child grow up in the American cultural world learns the words "dog", he will normally learn the cultural meaning of the word: the dog is "man's best friend", while a child brought up in the Chinese cultural world will be taught that the dog is a dirty and dangerous animal. These two kinds of 
minds made us have extremely different attitude at a same animal. People, who naturally associated the culture with their mother tongue, are naturally inclined to interpret things with their own cultural references. This natural inclination is called "intuitive competence".

A culture-specific phenomenon is thus one that is found to exist in a particular form or function in only one of the two cultures being compared. This doesn't mean that the phenomenon exists only in those particular cultures with another form. So in linguistic translation, it is important to be familiar with cultural background of the words.

In the famous work "A Dream of Red Mansion", there is such occasion: Wang Xifeng and Baoyu hold a funeral procession, and Wang said to Baoyu :"bie gei ta men hou zai ma shang." To show her shrewish and cordial vividly , David Hawkes translate this sentence into "you don't want to go clomping around the countryside like apes on horseback with those men", which reappear Wang Xifeng's tone lively.

\section{2 non-equivalence.}

It is apparent, and has been for a very long time indeed, that the ideal of total equivalence is a chimera. Languages are different from each other; they are different in form having distinct codes and rules regulating the construction of grammatical stretches of language and these forms have different meanings. In translating one invariably looks for an equivalent word to render the original meaning in the target language, using the word "equivalence" in this context immediately bring the idea that identify the absolute corresponding equivalent words in two languages. But this is not the case.

2.2.1 A term in one language does not have a counterpart in another language

American Historian Henry Brooks told us "words are slippery", he referred to the fact that language translation is difficult and subject to countless misinterpretations.

We often meet such circumstances in the course of translation: One thing is unique in one country, and the equivalent word reflecting this object can't be found in another country. Thus non-equivalence of words comes into being, and this illustrates cultural difference directly. For instance, we can't find equivalent Chinese term for "Sphinx's riddle"; on the other hand, "xia lian san fu, dong lian san jiu", which means urging people to exercise and keep fit, is difficult to be translated into English.

To shift from one language to another is by definition, to alter the forms. Further, the contrasting forms convey meanings which cannot but fail to coincide totally; there is no absolute synonymy between words in the same language, so we can't be surprised to discover a lack of synonymy between languages.

In that case, we sometimes use "loanword". When a word is adopted directly by one language from another, it is referred to as a loanword. Loanwords may retain a pronunciation similar to that of the original language. Such as some Chinese words: $k a$ fei (coffee), ji pu che (jeep), sha fa (sofa) etc, they have become the basic vocabulary in our daily life and nobody would feel "an exotic atmosphere" when using these words.

2.2.2 Words or terms in both languages that appear to refer to the same object or concept on the surface actually refer to quite different things.

First, we take an example to illustrate it. For the Chinese, ga li only means to be a kind of condiments. But the curry in England refers to the chicken, meat, or other food stewed of curry, and it is generally mixed with rice, for it is both salted and peppery. So curry $\neq$ ga li. Besides, there are many examples, about an object or concept that exists in one culture but not in another.

\subsection{Extension and Intension}

In different countries, when defining the same object, languages are different in extension and intension, which reflects cultural gaps, too.

2.3.1 Terms that have more or less the same primary meaning, but which have secondary or additional meanings that may differ considerably from each other.

We can see there are many color examples about it. In Chinese, blue implies pure and melancholy when it means salacious in English. And we find that we sometimes can't directly translate color words from one language to another without introducing subtle changes in meaning. The English phrase "red-blooded" doesn't mean "hong xue de",rather, it is another way of saying that someone or their behavior is confident and strong. And the English phrase "red-blooded" just means "having red eyes (for lack of sleep)", while the Chinese "equivalent" hong yan means at least two things: having red eyes, and interesting enough, "green" with envy.

2.3.2 Things or concepts that are represented by one or perhaps two terms in one language, but by many more terms in the other language.

In the broad sense, language is the symbolic representation of a person, and it compresses their historical and cultural 
backgrounds as well as their ways of living and thinking. For instance, the word "brother-in-law" is often used in our daily life, and it can be applied to my sister's husband, my husband's brothers and my husband's sisters' husbands, which in Chinese we called "mei fu", "xiao shu zi", "xiao jiu zi" and so on, when perceiving all of these relatives in a similar way. Language is not simply a means of reporting experience but, more important, it is the way of defining experience.

\subsection{Derivation}

Undoubtedly, culture is the forms of things that people have in mind, their models for perceiving, relating, and otherwise interpreting them. People often associate certain qualities with certain creatures or objects. These qualities often arouse certain reactions or emotions, although there may be little or even no scientific grounds for such association.

In the Chinese culture, the most-favored animals should be the dragon and phoenix. The dragon stands for the emperor when the phoenix stands for the empress. All the Chinese people regard themselves as descendants of the dragon. To English speaker, however, the dragon is often a symbols of evil, a fierce monster that destroys, and therefore must be destroyed. And the phoenix is by no means the spouse of a dragon; rather, it is associated with rebirth and resurrection.

The qualities that are associated, or the emotions that are aroused, are not always the same with different people.

The English word "daffodil" is often used to mean the spring and joy, (just like the "westwind"), as in Shakespeare's sonnet:

When daffodils begin to peer,

With heigh, the doxy over the dale!

Why, then comes in the sweet o' the year.

A deeper description of daffodils as the messenger of the spring can be found in William Wordsworth's "The Daffodils". Such an image of the daffodil being a symbol of the joyful spring time, however, is not popular in the Chinese literature. Conversely, a number of Chinese words with special images can hardly arise English-speaker's any association.

Another best example is “A Dream of Red Mansion”. Undoubtedly the title may cause misunderstanding in American's minds. For in their opinions, it means a person slept in a red room, which may cause their mysterious association. But conversely, it is not what the Chinese title means. David Hawkes's translation of "The Story of the Stone( another original Chinese title) is even easier for the American who don't have a solid foundation in ancient Chinese to understand the text. To this extent, it is a successful translation, for it is faithful to the original. However, to the Chinese, the title "A Dream of Red Mansion" can cause them unlimited association. This clearly illustrates cultural differences.

\section{The method on how to solve above translating problems}

For there are so many translating problems that be aroused by cultural gaps, how to deal with it is a very important process during translation. On the basis of his own translation practice Wang Zuoling stresses that in translating literature, one should emphasize the following three points: $1>$. to translate the concept and convey the complete feeling of the original rather than just one word; $2>$. to use different styles with different genres; and $3>$. to pay more attention to the reader. That is, a translator should attach greater importance to cultural equivalence than to any other aspects. Obviously, equivalence can be achieved, but not always on the same level. We can choose suitable translation method from the following six procedures of establishing cultural equivalence. It is the key point of translating for it can affect the translation very much.

\subsection{Retain original cultural flavor}

From the Jumplet's principles of literary translation,, we study that "A translation should read like an original". Whether to retain original cultural flavor depends on the literal form and inner meaning, which can be accepted by the reader.

We still cite the famous work "A Dream of Red Mansions" as the example.

Daiyu dao:"die le deng zhi qian ne, hai shi die le ren zhi qian? Ni you chuan bu guan mu ji zi. Na deng long jiao ta men qian tou dian zhe:zhe ge you qing qiao you liang, yuan shi yu li zi ji na zhe de.ni shou li zi ji na zhe zhe ge, qi bu hao? ming er zai song lai, --jiu shi le shou ye shi you xian de, zen me hu ran you bian chu zhe "pou fu cang zhu"de pi qi lai!"'(Cao xue qin<hong lou meng $>$ )

Daiyu said, "Which is more valuable, lamp or man? You're mot used to wearing pattens, so get them carry the horn lantern in front and take this one yourself, since it's handy and bright and meant to be used in the rain. Wouldn't that be better? You can send it back later. And even if you drop it, it won't matter what's come over you suddenly that you want to 'cut open your stomach to hide a pearl'?"(translated by Yang Xianyi and Gladys Yang).

"pou fu cang zhu" is translated into "cut open your stomach to hide a pearl" in literal meaning directly. The metaphor "to save a little only to lose a lot" in the original text is extremely lifelike in the translated text, which is widely 
accepted by the reader, and achieved the same effects as the English idiom "penny wise and pound foolish".

Then let us read three translation way of the sentence "A one-eyed person is the King of the Blind Kingdom":

\section{A. shan zhong wu lao hu, hou zi cheng dai wang; \\ B. shu zhong wu da jiang, liao hua chong xian feng; \\ C.mang ren guo li, du yan wei wang.}

In my opinion, the third one is the best answer. The first one is negative in Chinese culture, so it is no corresponding to the source language; let's read the second one, I think many foreigners don't know who Liao Hua is at all and somebody can't understand this allusion which is quoted from "Three Kingdoms" unless he knows this book very much. Therefore, the third one is the fittest one for it retains the original meaning and flavor.

Let me show you another example: "when he dined with his sister that evening, Madeline helped herself to a cigarette from his pack on the table, and lit and smoked it inexpertly. Her defiant, self-satisfied, somewhat pathetic air made Warren laugh. "When the cat's away, hey? He said." Ta gen ta mei mei na tian yi qi chi wan fan de shi hou, mei de lin cong zhuo shang ta de yan he li qu chu zhi xiang yan, dian le huo, bu tai zai hang de chou le qi lai. Ta na zhong jue jiang de, zi man de, you dian re ren lian ai de shen qi yin de hua lun haha da xiao. "mao bu zai le, hei!" ta shuo. Here "When the cat's away" is the abbreviation of the idiom "When the cat's away, the mice will play". The target language "mao bu zai le" keeps the abbreviation form of the original text, for the readers can understand the meaning according to the context: Warren and Madeline are sisters, when saying "When the cat's away", Warren just made a fun of his sister that she smoked furtively when their father is away. Other idioms such as "da cao jing she" (to stir up the grass and alert the snake), "xue zhong song tan"(to send charcoal in snowy weather), "hua she tian zu" (to draw a snake and add feet to it) have the same result.

From above examples, we can feel "retain original cultural flavor" is a good and effective way. It can maximize the features of the original style. It always is used in translating classical novel and idioms and so on, especially there are a complete story and have special context or circumstance. But not all translation adopt this way because different problems need different way to solve.

\subsection{Transliteration}

Transliteration can be divided into two main aspects: transliteration and transliteration plus a generic word.

\subsubsection{Transliteration}

Transliteration means words are translated according to the pronunciation, for instance, copy $(\mathrm{kao}$ bei), brandy(bai lan di), chocolate(qiao ke li), aspirin(a si pi lin); ma jiang(Mah--jong), kong zi(Confucius)etc. It often used to translate something unique in certain countries or areas, just like some food and games.

\subsubsection{Transliteration plus a generic word}

Sometimes, Chinese readers may not understand the explicit meaning when using transliteration for they have no chance to know the thing directly, so we should plus a generic word after transliteration if necessary. Such word include Motor(mo tuo che), ballet(ba lei wu), beer(pi jiu), Hamburger(han bao bao), Pizza(bi sa bing), AIDs(ai zi bing). The plus generic word can help us to know these "new" things clearly so that we won't make a joke.

Besides, transliteration and transliteration plus a generic word are used together such as Benz(ben chi, ben chi che), cigar(xue jia, xue jia yan)etc.

\subsection{Transference}

Because of some words are used widely in our daily life, maybe they are not original Chinese words we still can understand its meaning without translation. This method is called transference which means the original word is all transferred into the translated text wholly or partly. For instance: KTV, WTO, APEC, MTV, VCD etc.

\subsection{Replace the cultural expressions of the source text with those of the target language}

People in different regions use proverbs and sayings with their own distinct characteristics. Proverbs are short sayings of wisdom - of well-known maxims, truths or facts expressed succinctly and in a way that makes them easy to remember. And such method is most useful and helpful in translating proverbs. A large number of Chinese proverbs and sayings invariably display deep-rooted Chinese qualities. "zhi ma kai hua jie jie gao"(A sesame stalk puts forth blossoms notch by notch, higher and higher) is widely used by the Chinese people, who are familiar with or taught about the agriculture plant of sesame; "lu yao zhi ma li, ri jiu jian ren xin"(As a long road tests a horse's strength, so a long task proves a person's heart) "chu sheng niu du bu pa hu”(New-born calves make little of tigers) are also such examples.

These examples demonstrate the truth that people in different cultures do have the understanding of certain natural phenomena and social events. And they have a common wisdom with regard to many respects of life. This might 
explain why people say, "Great minds think alike", which in itself is a good example of the similarity in the two cultures.

\subsection{Translate the implied meaning}

Apparently, people in different cultures express words differently, because of difference of the mode of thinking. In that case, we should translate the implied meaning according to the context. For instance, "When I was in trouble, Paul was the only one who would stick his neck out to help me." Yi dan wo you le kun nan, bao luo shi wei yi yi ge gan mao feng xian lai bang zhu wo de ren. "I'm going straight" wo zai ye bu tou le (or, wo jue ding xi shou bu gan le).

\subsection{Explanation}

To explain is another good way to solve cultural gaps' problems. When translating certain words of the cultural flavor, there is no equivalent word in target language but only to explain. For example: Shylock refers to "lin se gui", spring chicken "nian qing de shao nv", go Dutch "ge fu ge de qian", Don't play uncle over me "bu yao dui wo shua wei feng", keep one's nose to the grindstone "mai tou ku gan" and so on. Another example "to carry coals to Newcastle " is translated into "yun mei dao niu ka si er, duo ci yi ju", which is clearly enough. Although, we have added "duo ci yi ju" to explain the metaphor in the sentence, the readers can't understand the word "Newcastle", we should illustrate that Newcastle is the centre of coals, carrying coals to that is superfluous.

\section{Conclusion}

The significance of translation is to transfer words and phrases from one language to another language. Briefly speaking, it is an art that retell the thought of author's accurately with a complete different language from original. In the light of definition above, we know that the original meaning of expression should be kept in version in translation, no addition, no deletion. Therefore, there are two factors in translation: accuracy and expressivity. A good interpreter needs special, highly developed skills to achieve the goal of translation that is to convey the meaning and style of the original language. This means that he must be skilled in more than vocabulary. He must also know the word's emotive aspects, as well as the culture's thought processes.

In this article, we just probe into the cultural gaps and the way to deal with the translating problems in this field. And as far as the cultural value of language is concerned, this paper only touches upon part of it. Culture-related problems about this aspect are complicated and difficult, and besides the issues that we have mentioned in this article, there're still lots of culture-related translation problems and methods, which leaves us much to learn.

\section{References}

Bell, Roger T. (2001). Translation and Translating: Theory and Practice. Shanghai foreign language education press.

Hickey, Leo. (2001). The Pragmatics of Translation. Shanghai foreign language education press.

Newmark, Peter. (2001). Approaches to Translation Shanghai foreign language education press.

Nord, Christiane. (2001). Translating As a Purposeful Activity. Shanghai foreign language education press.

Snell-Hornby \& Mary. (2001). Tranlation Studies. Shanghai foreign language education press.

Steiner, George. (2001). After Babel: Aspects of Language and Translation. Shanghai foreign language education press.

Wilss, Wolfram. (2001). The Science of Translation: Problems and Methods. Shanghai foreign language education press. 